\title{
Bisphenol analogs and their chlorinated derivatives in breast milk in China: occurrence and exposure assessment
}

Yumin Niu, Bin Wang, Runhui Yang, Yongning Wu, Yunfeng Zhao, Cuizhi Li, Jing Zhang, Yang Xing, Bing Shao *

Yumin Niu - Beijing Key Laboratory of Diagnostic and Traceability Technologies for Food Poisoning, Beijing Center for Disease Prevention and Control, Beijing, 100013, China

Bin Wang - Chinese Academy of Inspection and Quarantine Comprehensive Test Center, Beijing, 100123, China

Runhui Yang - College of Veterinary Medicine, China Agricultural University, Beijing, 100193, China

Yongning Wu - NHC Key Laboratory of Food Safety Risk Assessment, China National Center for Food Safety Risk Assessment, Beijing 100021, China

Yunfeng Zhao - NHC Key Laboratory of Food Safety Risk Assessment, China National Center for Food Safety Risk Assessment, Beijing 100021, China

Cuizhi Li - Inner Mongolia Yili Industrial Group Company Limited Share Ltd, Hohhot 010018, China

Jing Zhang - Beijing Key Laboratory of Diagnostic and Traceability Technologies for Food Poisoning, Beijing Center for Disease Prevention and Control, Beijing, 100013, China

Yang Xing - Beijing Key Laboratory of Diagnostic and Traceability Technologies for Food Poisoning, Beijing Center for Disease Prevention and Control, Beijing, 100013, China

Bing Shao - Beijing Key Laboratory of Diagnostic and Traceability Technologies for Food Poisoning, Beijing Center for Disease Prevention and Control, Beijing, 100013, China 
*Corresponding author: E-mail: shaobingch@sina.com

Tel: +86-10-64407191 


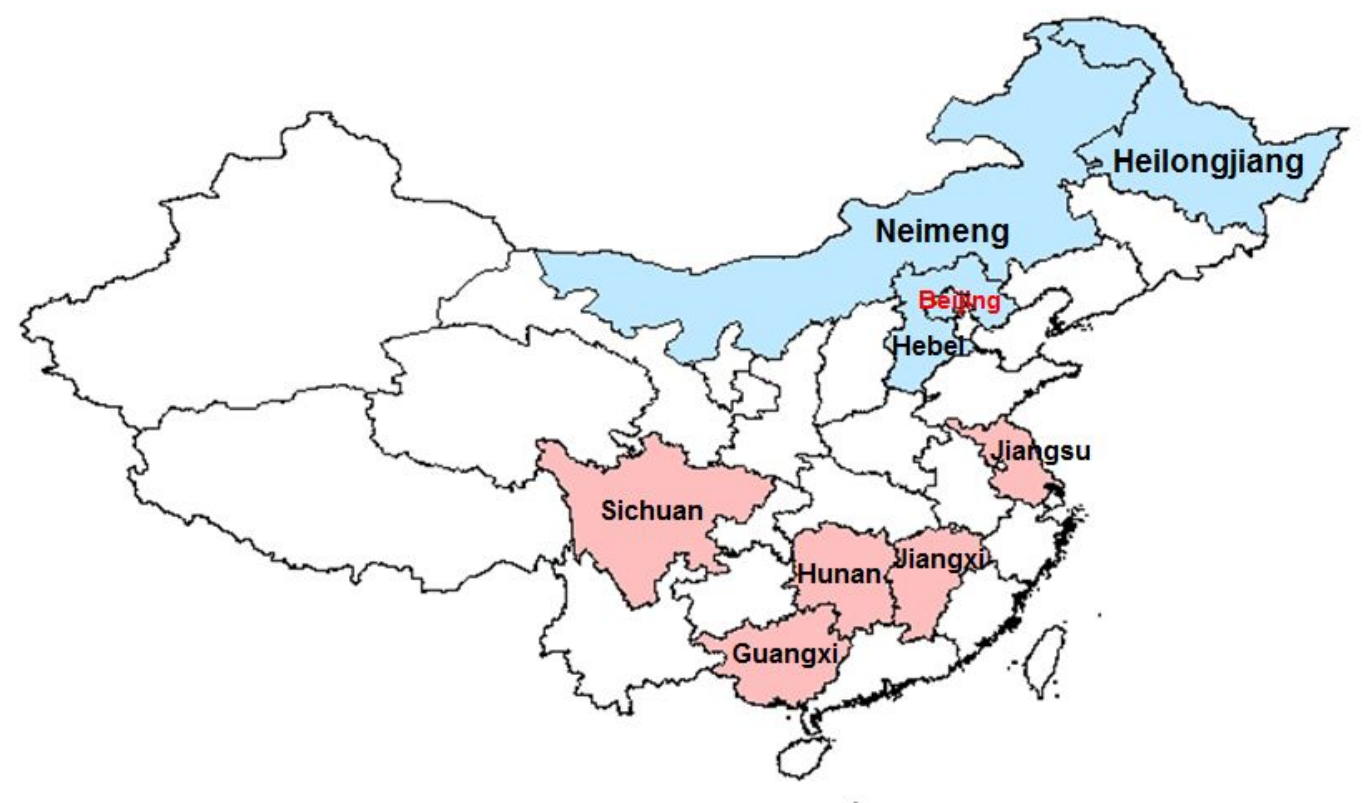

Figure S1. Sampling provinces in the Chinese human milk survey. 


\section{Chemical and Reagents}

BPA, BPB, BPF, BPS, BPAF and TCBPA were from Tokyo Chemical Industry (Tokyo, Japan). Other bisphenol analogues such as BPC, BPE, BPM, BPP, BPZ, BPAP, BPBP, BPFL and DHDPE were obtained from Sigma-Aldrich Inc. (St. Louis, MO). Chlorinated BPA including MCBPA, DCBPA and TriBPA were purchased from Toronto Research Chemical Int. (North York, Canada). Chlorinated BPF including MCBPF, DCBPF, TriBPF and TCBPF were synthesized in our previous study (Zheng et al., 2016). The isotope internal standards including BPA- ${ }^{13} \mathrm{C}_{12}, \mathrm{BPB}-{ }^{13} \mathrm{C}_{12}, \mathrm{BPF}-{ }^{13} \mathrm{C}_{12}$, BPS $-{ }^{13} \mathrm{C}_{12}$, BPAF- $\mathrm{d}_{4}$ and TCBPA- ${ }^{13} \mathrm{C}_{12}$ were all obtained from Cambridge Isotope Laboratories Inc. (Andover, MA). The purities of all compounds were more than $98 \%$. LC-MS grade water, methanol and acetonitrile were from Sigma-Aldrich (St. Louis, MO). HPLC grade of acetone and n-hexane were from Dikma Technologies Inc. (Lake Forest, CA). Formic acid (99\%) was purchased from Acros Organics (Morris Plains, NJ). Sodium bicarbonate (ACS grade) and sodium hydroxide $(98.5 \%)$ were obtained from J\&K Scientific Ltd. (Beijing, China). $\beta$-Glucuronidase/aryl-sulfatase mixture (extracted from Helix pomatia) was from Roche Diagnostics GmbH (Mannheim, Germany). Pyridine-3-sulfonyl chloride was obtained from Tokyo Chemical Industry (Tokyo, Japan). 
Table S1 The LOD and LOQ of individual BPs

\begin{tabular}{ccc}
\hline Compounds & $\mathrm{LOD}^{\mathrm{a}}\left(\mu \mathrm{g} \mathrm{L}^{-1}\right)$ & $\mathrm{LOQ}^{\mathrm{b}}\left(\mu \mathrm{g} \mathrm{L}^{-1}\right)$ \\
\hline BPA & 0.017 & 0.050 \\
BPB & 0.001 & 0.003 \\
BPC & 0.002 & 0.005 \\
BPE & 0.002 & 0.005 \\
BPF & 0.002 & 0.005 \\
BPM/BPP & 0.002 & 0.005 \\
BPS & 0.003 & 0.010 \\
BPZ & 0.003 & 0.010 \\
BPAF & 0.003 & 0.010 \\
BPAP & 0.002 & 0.005 \\
BPBP & 0.0003 & 0.001 \\
BPFL & 0.001 & 0.003 \\
DHDPE & 0.003 & 0.010 \\
MCBPA & 0.001 & 0.003 \\
DCBPA & 0.002 & 0.005 \\
TriCBPA & 0.002 & 0.005 \\
TCBPA & 0.002 & 0.005 \\
TBBPA & 0.002 & 0.005 \\
MCBPF & 0.017 & 0.050 \\
DCBPF & 0.067 & 0.200 \\
TriCBPF & 0.067 & 0.200 \\
TCBPF & 0.008 & 0.025 \\
\hline
\end{tabular}


Table S2 Comparison of BPA, BPF and BPS levels in breast milk samples with reported studies

\begin{tabular}{|c|c|c|c|c|c|c|c|c|c|c|c|c|c|c|c|}
\hline \multirow[t]{2}{*}{ Country } & \multirow{2}{*}{$\begin{array}{c}\text { Year of } \\
\text { collection }\end{array}$} & \multirow{2}{*}{$\begin{array}{c}\text { N. of } \\
\text { samples }\end{array}$} & \multicolumn{3}{|c|}{ BPA $(\mu \mathrm{g} / \mathrm{L}$ or $\mu \mathrm{g} / \mathrm{kg})$} & \multirow[b]{2}{*}{$\mathrm{DF}^{\mathrm{a}}(\%)$} & \multicolumn{4}{|c|}{$\mathrm{BPF}(\mu \mathrm{g} / \mathrm{L}$ or $\mu \mathrm{g} / \mathrm{kg})$} & \multicolumn{4}{|c|}{ BPS $(\mu \mathrm{g} / \mathrm{L}$ or $\mu \mathrm{g} / \mathrm{kg})$} & \multirow[t]{2}{*}{ Reference } \\
\hline & & & Mean & Median & Range & & Mean & Median & Range & DF (\%) & Mean & Median & Range & DF (\%) & \\
\hline Japan & - & 23 & 0.61 & 0.61 & $0.28-0.97$ & 100 & - & - & - & - & - & - & - & - & 1 \\
\hline Japan & - & 20 & 1.9 & 1.1 & $0.3-6.3$ & 90 & - & - & - & - & - & - & - & - & 2 \\
\hline Japan & $2000-2001$ & 101 & 3.41 & - & $1-7$ & 100 & - & - & - & - & - & - & - & - & 3 \\
\hline$J^{J a p a n}{ }^{b}$ & $2012-2013$ & 19 & 35.7 & 6.6 & $1.4-380$ & 100 & - & - & - & - & - & - & - & - & 4 \\
\hline USA & 2009-2010 & 30 & - & 1.3 & - & 100 & - & - & - & - & - & - & - & - & 5 \\
\hline USA & 2003-2006 & 21 & 3.13 & 0.68 & $\begin{array}{c}<0.22- \\
10.8\end{array}$ & 62 & - & - & - & - & - & - & - & - & 6 \\
\hline USA & $2006-2008$ & 23 & 2.1 & 0.8 & $<0.3-22.6$ & 75 & - & - & - & - & - & - & - & - & 7 \\
\hline Korea & - & 100 & - & 10.4 & $0.65-42.6$ & 100 & - & - & - & - & - & - & - & - & 8 \\
\hline Korea & 2011-2012 & 127 & - & 0.7 & $<0.3-43.2$ & 80 & - & - & - & - & - & - & - & - & 9 \\
\hline France & 2011 & 21 & 1.87 & 1.47 & $<0.09-6.12$ & 90 & - & - & - & - & - & - & - & - & 10 \\
\hline France & 2010 & 30 & 0.23 & 0.11 & $\begin{array}{c}<0.01- \\
1.16\end{array}$ & 87 & $\begin{array}{c}< \\
0.018\end{array}$ & $<0.018$ & $<0.018$ & 0 & 0.01 & $<0.001$ & $\begin{array}{c}<0.001- \\
0.23\end{array}$ & 3 & 11 \\
\hline Spain & - & 10 & - & - & $\begin{array}{c}<0.05- \\
11.5\end{array}$ & 60 & - & - & - & - & - & - & - & - & 12 \\
\hline Spain & 2015 & 100 & 1.6 & 0.26 & $<0.10-41$ & 83 & - & $<0.13$ & $<0.13-0.46$ & $22(\mathrm{n}=91)$ & $<0.25$ & $<0.25$ & $\begin{array}{c}<0.25- \\
0.37\end{array}$ & $1(\mathrm{n}=91)$ & 13 \\
\hline Canada & 2009-2011 & 56 & $<0.2$ & $<0.2$ & $<0.2-1.9$ & 5 & - & - & - & - & - & - & - & - & 14 \\
\hline Canada & 2009-2011 & 278 & - & 0.11 & 0.65 & 26 & - & - & - & - & - & - & - & - & 15 \\
\hline China & 2014 & 20 & 0.136 & 0.074 & $\begin{array}{c}<0.05- \\
0.548\end{array}$ & 85 & 0.031 & 0.014 & $\begin{array}{c}<0.010- \\
0.166\end{array}$ & 60 & 0.034 & $<0.010$ & $\begin{array}{c}<0.010- \\
0.683\end{array}$ & 5 & 16 \\
\hline China & 2018-2019 & 190 & 2.5 & 0.21 & $<0.20-15$ & 53 & $\begin{array}{c}< \\
0.37\end{array}$ & $<0.37$ & $<0.37$ & 0 & 0.19 & $<0.10$ & $\begin{array}{c}<0.10- \\
1.3\end{array}$ & 44 & 17 \\
\hline
\end{tabular}




\begin{tabular}{|c|c|c|c|c|c|c|c|c|c|c|c|c|c|c|c|}
\hline China & 2014 & 181 & 0.444 & 0.255 & $\begin{array}{c}<0.050- \\
5.861\end{array}$ & 73 & 0.107 & 0.051 & $\begin{array}{c}<0.001- \\
3.716\end{array}$ & 82 & 0.027 & $<0.003$ & $\begin{array}{c}<0.003- \\
0.453\end{array}$ & 46 & This study \\
\hline
\end{tabular}

a detect frequency

${ }^{b} \mathrm{ng} / \mathrm{g}$ 
References:

1. Sun, Y.; Irie, M.; Kishikawa, N.; Wada, M.; Kuroda, N.; Nakashima, K., Determination of bisphenol $\mathrm{A}$ in human breast milk by HPLC with column-switching and fluorescence detection. Biomedical Chromatography 2004, 18, 501-507.

2. Ye, X. Y.; Kuklenyik, Z.; Needham, L. L.; Calafat, A. M., Measuring environmental phenols and chlorinated organic chemicals in breast milk using automated on-line column-switchinghigh performance liquid chromatography-isotope dilution tandem mass spectrometry. Journal of Chromatography B-Analytical Technologies in the Biomedical and Life Sciences 2006, 831, $110-115$.

3. Kuruto-Niwa, R.; Tateoka, Y.; Usuki, Y.; Nozawa, R., Measurement of bisphenol A concentrations in human colostrum. Chemosphere 2007, 66, 1160-1164.

4. Nakao, T.; Akiyama, E.; Kakutani, H.; Mizuno, A.; Aozasa, O.; Akai, Y.; Ohta, S., Levels of Tetrabromobisphenol A, Tribromobisphenol A, Dibromobisphenol A, Monobromobisphenol A, and Bisphenol A in Japanese Breast Milk. Chemical Research in Toxicology 2015, 28, 722728.

5. Duty, S. M.; Mendonca, K.; Hauser, R.; Calafat, A. M.; Ye, X.; Meeker, J. D.; Ackerman, R.; Cullinane, J.; Faller, J.; Ringer, S., Potential Sources of Bisphenol A in the Neonatal Intensive Care Unit. Pediatrics 2013, 131, 483-489.

6. Zimmers, S. M.; Browne, E. P.; O'Keefe, P. W.; Anderton, D. L.; Kramer, L.; Reckhow, D. A.; Arcaro, K. F., Determination of free Bisphenol A (BPA) concentrations in breast milk of US women using a sensitive LC/MS/MS method. Chemosphere 2014, 104, 237-243.

7. Mendonca, K.; Hauser, R.; Calafat, A. M.; Arbuckle, T. E.; Duty, S. M., Bisphenol A 
concentrations in maternal breast milk and infant urine. International Archives of Occupational and Environmental Health 2014, 87, 13-20.

8. Yi, B.; Kim, C.; Yang, M., Biological monitoring of bisphenol A with HLPC/FLD and LC/MS/MS assays. Journal of Chromatography B-Analytical Technologies in the Biomedical and Life Sciences 2010, 878, 2606-2610.

9. Lee, J.; Choi, K.; Park, J.; Moon, H. B.; Choi, G.; Lee, J. J.; Suh, E.; Kim, H. J.; Eun, S. H.; Kim, G. H.; Cho, G.; Kim, S. K.; Kim, S.; Kim, S. Y.; Kim, S.; Eom, S.; Choi, S.; Kim, Y. D.; Kim, S., Bisphenol A distribution in serum, urine, placenta, breast milk, and umbilical cord serum in a birth panel of mother-neonate pairs. Science of the Total Environment 2018, 626, 1494-1501. 10. Migeot, V.; Dupuis, A.; Cariot, A.; Albouy-Llaty, M.; Pierre, F.; Rabouan, S., Bisphenol A and Its Chlorinated Derivatives in Human Colostrum. Environmental Science \& Technology 2013, 47, 13791-13797.

11. Deceuninck, Y.; Bichon, E.; Marchand, P.; Boquien, C. Y.; Legrand, A.; Boscher, C.; Antignac, J. P.; Le Bizec, B., Determination of bisphenol A and related substitutes/analogues in human breast milk using gas chromatography-tandem mass spectrometry. Analytical and Bioanalytical Chemistry 2015, 407, 2485-2497.

12. Rodriguez-Gomez, R.; Jimenez-Diaz, I.; Zafra-Gomez, A.; Ballesteros, O.; Navalon, A., A multiresidue method for the determination of selected endocrine disrupting chemicals in human breast milk based on a simple extraction procedure. Talanta 2014, 130, 561-570.

13. Dualde, P.; Pardo, O.; Corpas-Burgos, F.; Kuligowski, J.; Gormaz, M.; Vento, M.; Pastor, A.; Yusa, V., Biomonitoring of bisphenols A, F, Sin human milk and probabilistic risk assessment for breastfed infants. Science of the Total Environment 2019, 668, 797-805. 
14. Arbuckle, T. E.; Weiss, L.; Fisher, M.; Hauser, R.; Dumas, P.; Berube, R.; Neisa, A.; LeBlanc, A.; Lang, C.; Ayotte, P.; Walker, M.; Feeley, M.; Koniecki, D.; Tawagi, G., Maternal and infant exposure to environmental phenols as measured in multiple biological matrices. Science of the Total Environment 2015, 508, 575-584.

15. Cao, X. L.; Popovic, S.; Arbuckle, T. E.; Fraser, W. D., Determination of free and total bisphenol A in human milk samples from Canadian women using a sensitive and selective GCMS method. Food Additives and Contaminants Part a-Chemistry Analysis Control Exposure \& Risk Assessment 2015, 32, 120-125.

16. Niu, Y. M.; Wang, B.; Zhao, Y. F.; Zhang, J.; Shao, B., Highly Sensitive and HighThroughput Method for the Analysis of Bisphenol Analogues and Their Halogenated Derivatives in Breast Milk. Journal of Agricultural and Food Chemistry 2017, 65, 10452-10463. 17. Jin, H.; Xie, J.; Mao, L.; Zhao, M.; Bai, X.; Wen, J.; Shen, T.; Wu, P., Bisphenol analogue concentrations in human breast milk and their associations with postnatal infant growth. Environmental Pollution 2020, 259, 113779. 